\title{
Comparison of i-gel ${ }^{\circledR}$ and LMA Supreme ${ }^{\circledR}$ during laparoscopic cholecystectomy
}

\author{
Sang Yoong Park, Jong Cheol Rim, Hyuk Kim, Ji Hyeon Lee, and \\ Chan Jong Chung
}

Department of Anesthesiology and Pain Medicine, Dong-A University College of Medicine, Busan, Korea

\begin{abstract}
Background: In laparoscopic surgical procedures, many clinicians recommend supraglottic airway devices as good alternatives to intubation. We compared the i-gel ${ }^{\circledR}$ (i-gel) and LMA Supreme ${ }^{\circledR}$ (Supreme Laryngeal Mask Airway, SLMA) airway devices during laparoscopic cholecystectomy regarding sealing pressure and respiratory parameters before, during, and after pneumoperitoneum.

Methods: Following Institutional Review Board approval and written informed consent, 93 patients were randomly allocated into the i-gel $(n=47)$ or SLMA group $(n=46)$. Insertion time, number of insertion attempts, and fiberoptic view of glottis were recorded. Oropharyngeal leak pressure (OLP), the use of airway manipulation, peak inspiratory pressure, lung compliance, and hemodynamic parameters were measured before, during, and after pneumoperitoneum.

Results: There were no significant differences between the two groups regarding demographic data, insertion time, fiberoptic view of glottis, and the use of airway manipulation. The gastric tube insertion time was longer in the i-gel group (20.4 $\pm 3.9 \mathrm{~s})$ than in the SLMA group $(16.7 \pm 1.6 \mathrm{~s})(\mathrm{P}<0.001)$. All devices were inserted on the first attempt, excluding one case in each group. Peak inspiratory pressure, lung compliance, and OLP changed following carbon dioxide pneumoperitoneum in each group, but there were no significant differences between the groups.

Conclusions: Both the i-gel and SLMA airway devices can be comparably used in patients who undergo laparoscopic cholecystectomy, and they offer similar performance including OLP.
\end{abstract}

Key Words: I-gel, Laparoscopic cholecystectomy, Supreme laryngeal mask airway.

\section{Introduction}

Airway management has become more refined with the in-

Corresponding author: Chan Jong Chung, M.D., Ph.D.

Department of Anesthesiology and Pain Medicine, Dong-A University College of Medicine, 26, Daeshingongwon-ro, Seo-gu, Busan 49201, Korea

Tel: 82-51-240-5390, Fax: 82-51-247-7819

E-mail: cjchung@dau.ac.kr

Received: March 5, 2015.

Revised: 1st, May 22, 2015; 2nd, June 3, 2015.

Accepted: June 10, 2015.

Korean J Anesthesiol 2015 October 68(5): 455-461

http://dx.doi.org/10.4097/kjae.2015.68.5.455 troduction of many airway devices. In the past several decades, a variety of supraglottic airway devices (SADs) have been introduced with the goal of a more convenient replacement of tracheal intubation. The advantages of SADs include easy insertion, favorable respiratory mechanics, stable hemodynamics, and decreased airway morbidity [1].

The i-gel ${ }^{\circledR}$ (Intersurgical, Wokingham, UK) is a latex-free SAD with a non-inflatable cuff and medical-grade thermoplastic elastomer. The design creates a more intimate interface for interacting with supraglottic tissue [2]. The i-gel offers a good seal during anesthesia for both controlled ventilation and spontaneous breathing [3-5].

The LMA Supreme ${ }^{\circledR}$ device (Supreme Laryngeal Mask Airway [SLMA], The Laryngeal Mask Company, St. Helier, Jersey, $\mathrm{UK}$ ) consists of a curved and rigid airway tube, a drain tube

(c) This is an open-access article distributed under the terms of the Creative Commons Attribution Non-Commercial License (http://creativecommons.org/ licenses/by-nc/4.0/), which permits unrestricted non-commercial use, distribution, and reproduction in any medium, provided the original work is properly cited. 
placed within the center of the airway, and a relatively large inflatable cuff made of medical-grade plastic (polyvinyl chloride) that provides high airway leak pressure.

There has been significant interest in these two devices. A number of studies have been conducted in response to concerns regarding their safety and effectiveness in various situations [6-8].

Changes in respiratory mechanics following pneumoperitoneum, during a laparoscopic cholecystectomy, may result in increased airway pressures that may exceed the oropharyngeal leak pressure (OLP) of the device being used. The increased intra-abdominal pressure from pneumoperitoneum requires a higher airway pressure for adequate pulmonary ventilation, for which the i-gel and SLMA devices were designed. Laparoscopic surgery provides the harshest environment for assessing the efficacy of SADs during positive pressure ventilation [9].

The primary goal of the present study was to compare the i-gel and SLMA devices for patients undergoing elective laparoscopic cholecystectomy regarding the OLP before, during, and after pneumoperitoneum.

\section{Materials and Methods}

The prospective study was conducted after obtaining approval from the Institutional Review Board (approval date: $12 / 18 / 2013$ ), and the study was registered at cris.nih.go.kr. A written informed consent for participation in the study was obtained from each patient. The study involved 100 adult patients with American Society of Anesthesiologists (ASA) physical status I or II who were scheduled to undergo elective laparoscopic cholecystectomy under general anesthesia. Pregnancy, gastric reflex, and suspected airway difficulty were grounds for exclusion. The patients were randomly allocated into the i-gel or SLMA group (50 patients in each group) using computer-generated numbers.

Premedication was not provided. In the operating room, the usual vital signs were monitored, and a bispectral index (BIS; Aspect Medical Systems, Newton, MA, USA) sensor was applied before induction. The recorded baseline data were: electrocardiogram, noninvasive blood pressure, and oxyhemoglobin saturation.

All patients were in a neutral position with oxygen supplied by a face mask at a flow rate of $8 \mathrm{~L} / \mathrm{min}$. After preoxygenation, target-controlled infusion (TCI) of propofol with an effect-site concentration (Ce) of $4 \mu \mathrm{g} / \mathrm{ml}$ and TCI of remifentanil with a Ce of $5 \mathrm{ng} / \mathrm{ml}$ were started to induce anesthesia using an Orchestra $^{\circledR}$ Base Primea device (Fresenius Kabi, Brezins, France). After confirming a lack of response to voice, loss of eyelid reflex, and BIS $<50$, a neuromuscular blockade was achieved with rocuronium $0.8 \mathrm{mg} / \mathrm{kg}$. Jaw relaxation, the absence of movement, and full muscle relaxation were achieved. The SAD was inserted by the same anesthesiologist, who had performed more than 50 insertions of both the i-gel and SLMA devices. The selected size of the SAD depended on patient weight, in accordance with the manufacturer's recommendations, as follows: i-gel sizes 3, 4, and 5 for patients weighing $<50,50-90$, and $>90 \mathrm{~kg}$, respectively; and SLMA sizes 3, 4, and 5 for patients weighing $<50,50-70$, and $70-100 \mathrm{~kg}$, respectively.

Effective ventilation was confirmed via the visualization of end-tidal carbon dioxide $\left(\mathrm{ETCO}_{2}\right)$ square waveforms and bilateral chest movements on manual ventilation. If there was airway obstruction or a critical air leakage, then the device was removed, and a different sized device was reinserted. If the insertion of an SAD required more than four attempts, it was considered a failure, and a tracheal tube was inserted. The insertion time of the device was defined as the time between retrieving the device and visualization of the first $\mathrm{ETCO}_{2}$ waveforms. After SLMA insertion, the cuff was inflated to a pressure of 60 $\mathrm{cmH}_{2} \mathrm{O}$, using a cuff pressure monitor (Mallinckrodt Medical, Athlone, Ireland) and maintained at less than $60 \mathrm{cmH}_{2} \mathrm{O}$ [26]. A circle anesthesia breathing system was connected (inspired tidal volume $8 \mathrm{ml} / \mathrm{kg}$, inspiratory:expiratory ratio of $1: 2$, and respiratory rate of 10 breaths/min under a fresh gas flow rate of $3 \mathrm{~L} / \mathrm{min}$ ). Effective ventilation was defined as a square-wave capnograph trace with $\mathrm{ETCO}_{2}$ values of $30-45 \mathrm{cmH}_{2} \mathrm{O}$ and normal thoracoabdominal movements.

A gastric tube was placed through the gastric vent tube of the device (size: 12 Fr for the i-gel group and 14 Fr for the SLMA group). The gastric tubes were lubricated with a water-soluble lubricant. The insertion time of the gastric tube was recorded. The correct placement of the gastric tube was affirmed with epigastric auscultation by the penetration of inputted air. The airway manipulations required were: neck extension and flexion, jaw thrust, or a chin lift when an air leak sound was detected.

After appropriate placement of the SAD, the OLP was recorded at $1 \mathrm{~min}$ after induction (TO1), $5 \mathrm{~min}$ after the start of $\mathrm{CO}_{2}$ pneumoperitoneum (TO2), and $5 \mathrm{~min}$ after the removal of pneumoperitoneum (TO3). The OLP was measured by closing the adjustable pressure limiting valve of the circle system at a fixed gas flow of $3 \mathrm{~L} / \mathrm{min}$, and noting the airway pressure (maximum allowed was $40 \mathrm{cmH}_{2} \mathrm{O}$ ) at which equilibrium was reached or when there was an audible gas leakage from the throat by an auscultation of stethoscope. A flexible fiberoptic scope (external diameter $3.1 \mathrm{~mm}$; Olympus Optical, Tokyo, Japan) was inserted into the airway tube of the two devices to permit viewing of the laryngeal structures. The fiberoptic view of glottis was graded using the following scoring system: 1, vocal cords not seen; 2 , vocal cords plus anterior epiglottis visible; 3 , vocal cords plus posterior epiglottis visible; and 4, vocal cords only [10].

The Ce of TCI-propofol was adjusted to maintain a BIS of $45-55$, and that of TCI-remifentanil was adjusted to maintain 
blood pressure within $\pm 20 \%$ of the baseline value. Rocuronium $0.1 \mathrm{mg} / \mathrm{kg}$ was administered under peripheral neuromuscular monitoring guidance. At the end of surgery, pyridostigmine 0.2 $\mathrm{mg} / \mathrm{kg}$ and glycopyrrolate $0.01 \mathrm{mg} / \mathrm{kg}$ were used to reverse the effects of rocuronium.

Heart rate, systolic blood pressure, and diastolic blood pressure were recorded at baseline (T0), 1 min after induction (T1), 5 min before $\mathrm{CO}_{2}$ pneumoperitoneum (T2), 5 min after the start of $\mathrm{CO}_{2}$ pneumoperitoneum (T3), $5 \mathrm{~min}$ before the removal of pneumoperitoneum (T4), and 5 min after the removal of pneumoperitoneum (T5). The peak inspiratory pressure (PIP) and pulmonary compliance were recorded and compared between the groups at T1-5 using the spirometer of a Primus Dräger anesthesia ventilator (Dräger Medical GmbH, Lübeck, Germany). The peritoneal insufflation pressure was set at $15 \mathrm{mmHg}$ [11], and the reverse Trendelenburg position was limited to 30 degrees.

The device was removed when the patient started responding to verbal commands. Complications were investigated, such as blood on the device or a postoperative sore throat at $1 \mathrm{~h}$.

\section{Statistical analysis}

The sample size was based on data from a pilot study of 20 patients in the SLMA group, in which the OLP was measured $\left(27.0 \pm 4.5 \mathrm{cmH}_{2} \mathrm{O}\right)$ and compared with previous data [11]. To detect a difference of $10 \%$ in the OLP between the groups with a two-tailed $\alpha=0.05$ and a power of $80 \%$, a sample size of 44 patients would be required. There was the recruitment of $50 \mathrm{pa}-$ tients per group to allow for potential dropout of subjects.

Data were recorded using a data collection sheet and analyzed using a Microsoft Excel spreadsheet and SPSS version 18 (SPSS, Chicago, IL, USA). Statistical comparisons between the SADs were made using Student's t-test or Mann-Whitney's U test for continuous data and the chi-squared or Fisher's exact test for categorical data. Data are presented as the mean \pm SD or number of patients (\%). A P value $<0.05$ was considered statistically significant.

\section{Results}

One hundred patients were included in the study between December 2013 and March 2014. Seven patients were excluded, three in the i-gel group and four in the SLMA group, because the laparoscopic cholecystectomy was converted to open cholecystectomy during surgery (Fig. 1). Age, sex, ASA physical status, height, weight, anesthesia time, and pneumoperitoneum time were not statistically significant between the groups (Table 1).

Table 2 summarizes the comparative data and complications for the i-gel and SLMA groups. The i-gel sizes of 3, 4, and 5 were used for $5(10.6 \%), 42(89.4 \%)$, and 0 patients $(0 \%)$, respective-

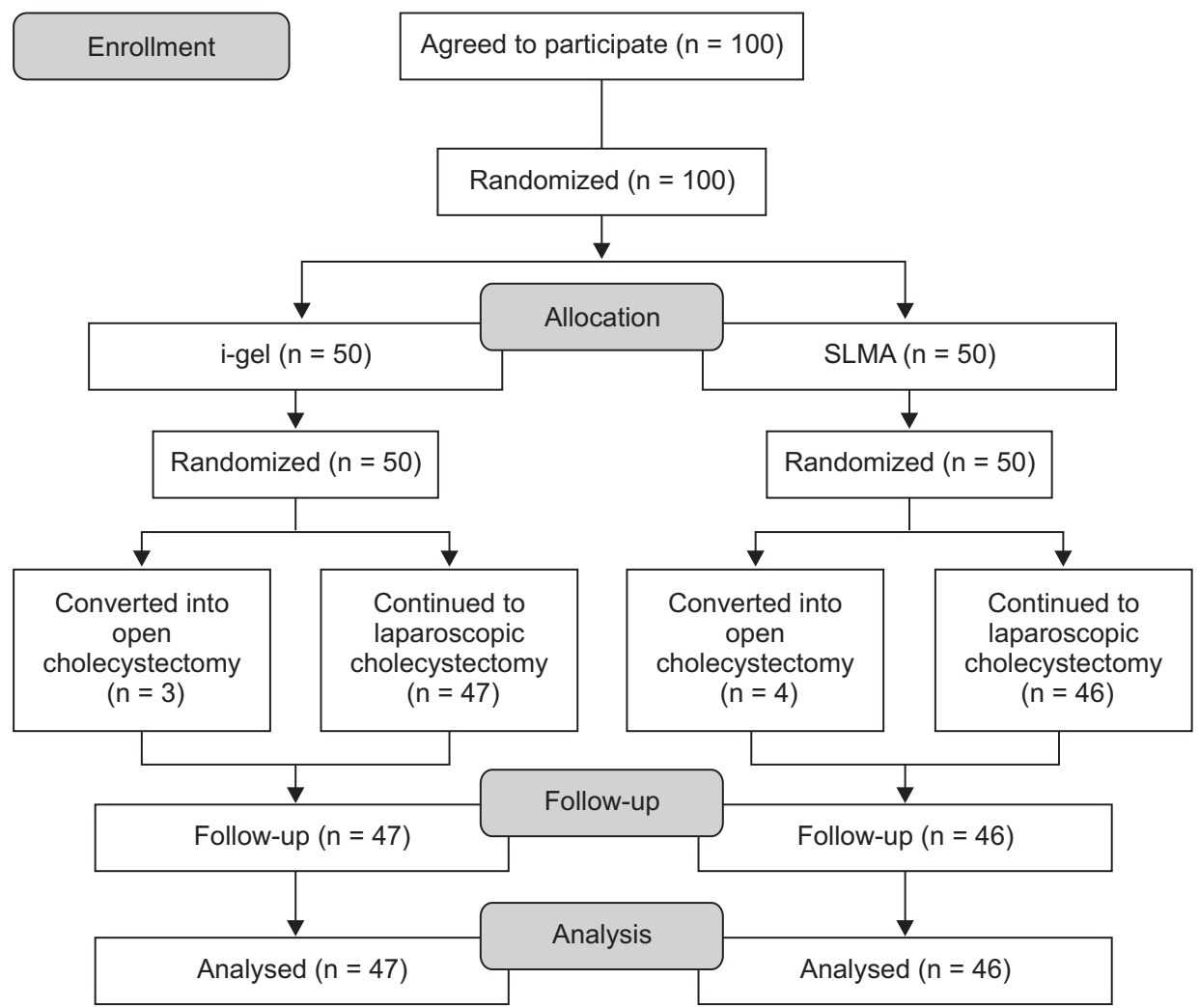

Fig. 1. CONSORT flow diagram for the study patients. 
Table 1. Patients' Demographic Characteristics and Anesthetic Properties

\begin{tabular}{lccc}
\hline & i-gel $(\mathrm{n}=47)$ & SLMA $(\mathrm{n}=46)$ & P \\
\hline Age $(\mathrm{yr})$ & $53.8 \pm 12.1$ & $55.9 \pm 14.9$ & 0.45 \\
Sex (M/F) & $21 / 26$ & $25 / 21$ & 0.23 \\
ASA I/II/III & $9 / 24 / 14$ & $9 / 24 / 13$ & 0.99 \\
Height (cm) & $163.6 \pm 7.4$ & $162.7 \pm 7.6$ & 0.55 \\
Weight (kg) & $64.3 \pm 8.9$ & $64.1 \pm 11.1$ & 0.93 \\
Anesthesia time (min) & $77.0 \pm 17.9$ & $72.0 \pm 17.0$ & 0.17 \\
Pneumoperitoneum time (min) & $36.1 \pm 14.6$ & $32.3 \pm 15.3$ & 0.22 \\
\hline
\end{tabular}

Data are presented as mean \pm standard deviation, or numbers. ASA: American Society of Anesthesiologist, SLMA: Supreme laryngeal mask airway.

Table 2. Comparative Data and Complications on Airway Devices

\begin{tabular}{lccc}
\hline & i-gel $(\mathrm{n}=47)$ & SLMA $(\mathrm{n}=46)$ & P \\
\hline Size $(3 / 4 / 5)$ & $5 / 42 / 0$ & $7 / 35 / 4$ & 0.08 \\
Attempt No. $(1 / 2 / 3)$ & $46 / 1 / 0$ & $45 / 0 / 1$ & 0.37 \\
Insertion time (sec) & $15.7 \pm 3.7$ & $15.7 \pm 3.1$ & 0.99 \\
Fiberoptic view (1/2/3/4) & $0 / 3 / 16 / 28$ & $1 / 9 / 10 / 26$ & 0.14 \\
Gastric tube insertion time (sec) & $20.4 \pm 3.9$ & $16.7 \pm 1.6$ & $<0.001$ \\
Audible air leak & 5 & 8 & 0.39 \\
Manipulation frequency (0/1/2) & $43 / 4 / 0$ & $39 / 6 / 1$ & 0.45 \\
Complications & & & 0.51 \\
$\quad$ Blood on device & 3 & 2 & 0.19 \\
Sore throat & 4 & 1 & 1.00 \\
$\quad$ Others & 2 & 2 & \\
\hline
\end{tabular}

Data are presented as mean \pm standard deviation, or numbers. Fiberoptic view: 1 , vocal cords not seen; 2 , vocal cords plus anterior epiglottis; 3 , vocal cords plus posterior epiglottis; 4, vocal cords only. SLMA: Supreme laryngeal mask airway.
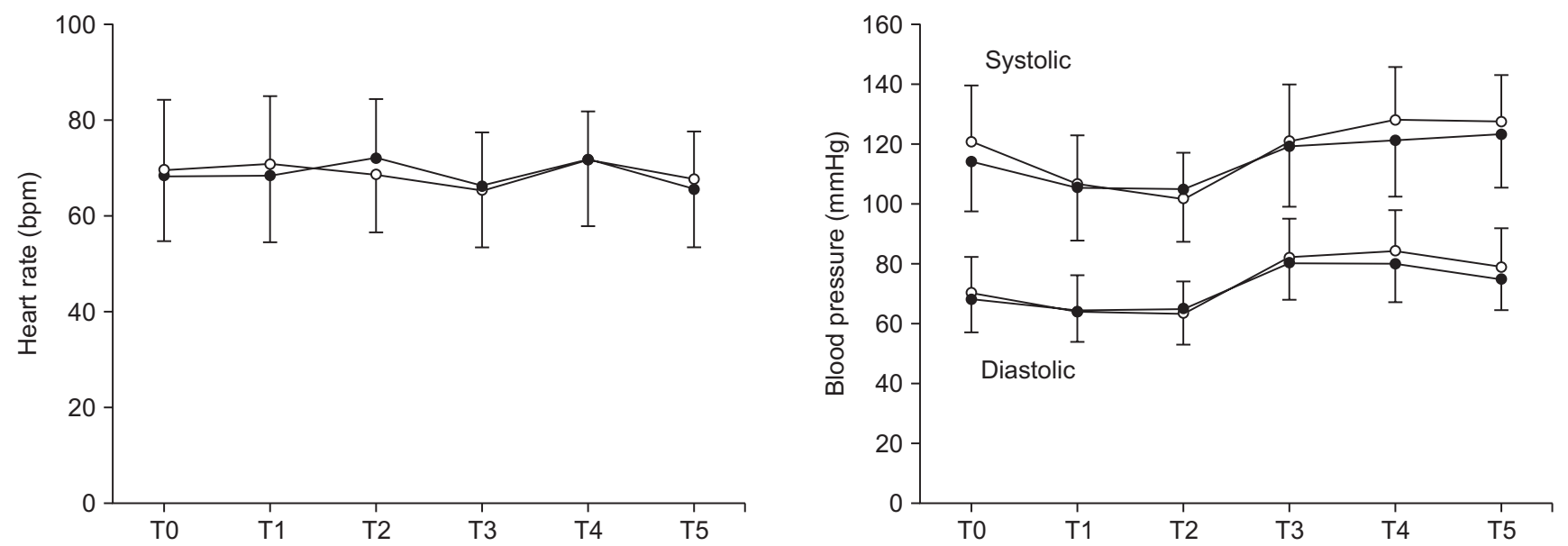

Fig. 2. Changes of heart rate and blood pressure between the i-gel group $(\circ)$ and the SLMA group $(\bullet)$. Values are mean \pm SD. There was no significant difference in heart rate and blood pressure between the groups. T0: baseline, T1: $1 \mathrm{~min}$ after induction, T2: 5 min before $\mathrm{CO}_{2}$ pneumoperitoneum, T3: 5 min after start of $\mathrm{CO}_{2}$ pneumoperitoneum, T4: 5 min before removal of pneumoperitoneum, T5: 5 min after removal of pneumoperitoneum.

ly. The SLMA sizes of 3, 4, and 5 were used for 7 (15.2\%), 35 (76.1 $\%)$, and 4 patients (8.7\%), respectively. All the devices were inserted on the first attempt, with the exception of one i-gel device that required a second insertion attempt and one SLMA device that required three attempts. There was no significant difference between the groups concerning insertion time (i-gel vs. SLMA:
$15.7 \pm 3.7 \mathrm{~s}$ vs. $15.7 \pm 3.1 \mathrm{~s} ; \mathrm{P}=0.99)$. The fiberoptic view of glottis did not differ significantly between the groups $(\mathrm{P}=0.14)$. A significantly longer gastric tube insertion time was noted in the i-gel group (20.4 $\pm 3.9 \mathrm{~s})$ than in the SLMA group (16.7 $\pm 1.6 \mathrm{~s})(\mathrm{P}$ $<0.001)$. Both heart rate and blood pressure did not differ at any measurement times (T0, T1, T2, T3, T4, and T5) between the 
groups (Fig. 2).

During pneumoperitoneum, an audible air leak occurred with five patients in the i-gel group and eight patients in the SLMA group. Airway manipulations (e.g., pushing and pulling of the device, jaw thrust, chin lift, neck extension, or flexion) were required in four patients in the i-gel group and seven patients in the SLMA group.

The OLP was lower after the induction period (TO1) than during the $\mathrm{CO}_{2}$ pneumoperitoneum period (TO2) and after the pneumoperitoneum period (TO3) in each group; however, the OLP was not significantly different in any measurement period between the two groups (Fig. 3).

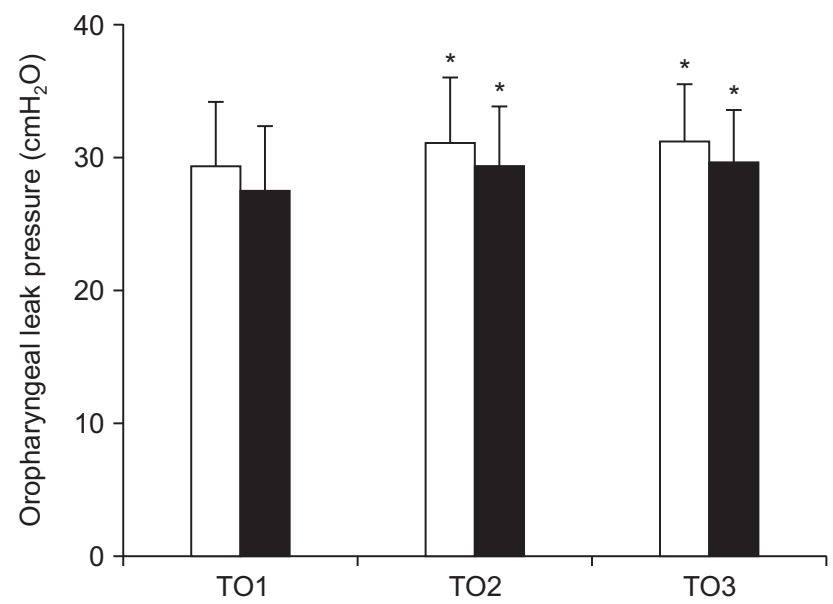

Fig. 3. Changes of oropharyngeal leak pressure between both i-gel ( $\square$ ) and SLMA ( $)$ groups at various points. Data are represented as the mean \pm SD. $* \mathrm{P}<0.01$ compared with TO1 in each group. TO1: 1 min after induction, TO2: 5 min after start of $\mathrm{CO}_{2}$ pneumoperitoneum and TO3: 5 min after removal of pneumoperitoneum.

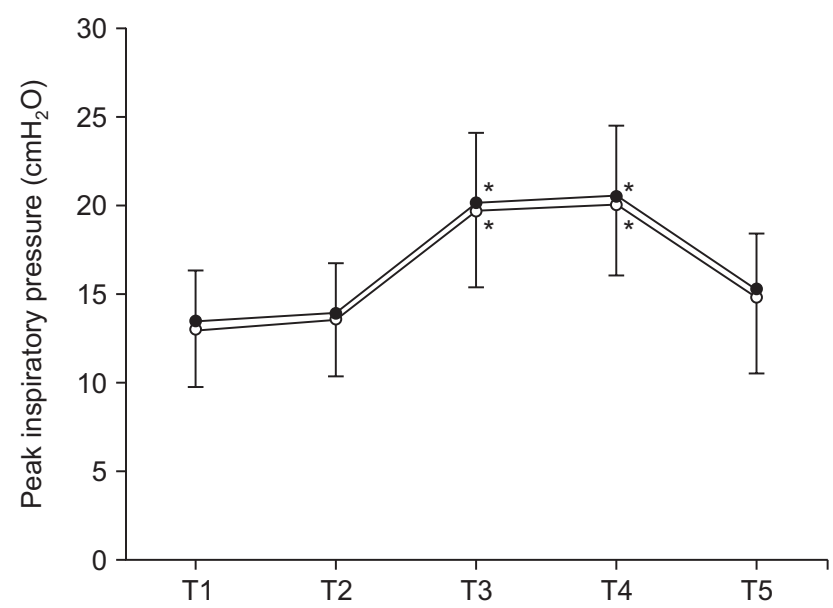

In both groups, the PIP was higher and pulmonary compliance was significantly lower during pneumoperitoneum (T3, T4) than before pneumoperitoneum (T2). However, the PIP and pulmonary compliance did not differ at any measurement times (T1, T2, T3, T4, and T5) between the groups (Fig. 4).

There was no significant difference in the incidence of postoperative complications between the groups. Blood on the device was observed concerning three patients in the i-gel group and two patients in the SLMA group. Four patients in the i-gel group and one patient in the SLMA group developed a postoperative sore throat.

\section{Discussion}

The present clinical study illustrated that both the i-gel and SLMA devices were used successfully to secure a good airway with a low rate of complications for a laparoscopic cholecystectomy. The OLPs of the i-gel and SLMA groups were nearly identical before, during, and after $\mathrm{CO}_{2}$ pneumoperitoneum. The SLMA device was associated with a shorter gastric tube insertion time than the i-gel device. There were no significant differences found for attempt number, SAD insertion time, or other complications.

The i-gel and SLMA insertions were successful on the first attempt in all but one patient in each group. The mean insertion times of the i-gel and SLMA devices were not statistically significantly different. Despite the time required for insufflation of the SLMA cuff, the insertion times of the groups were similar. These results are comparable with those of a recent study [12] that compared the i-gel and LMA Classic in children. Recently, Chen et al. [13] reported a meta-analysis that included 10 stud-

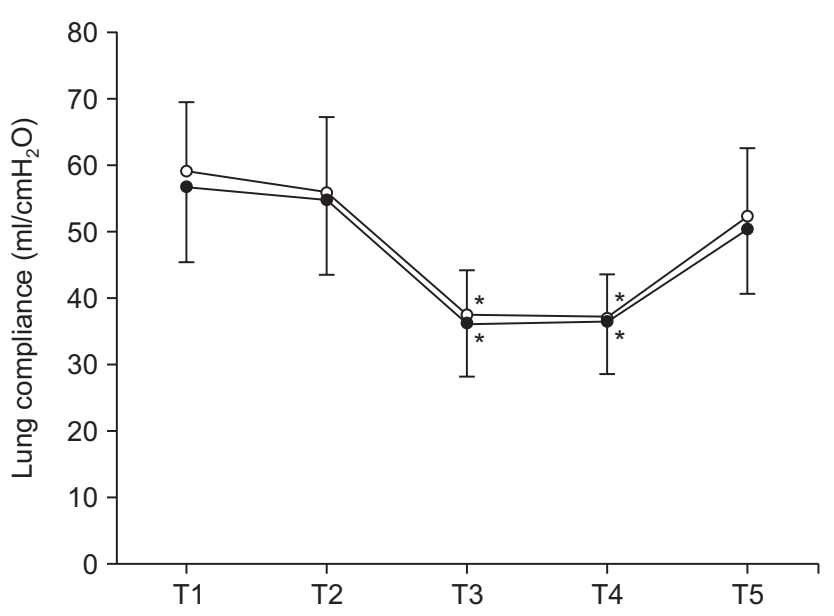

Fig. 4. Changes of peak inspiratory pressure and lung compliance between the i-gel group ( $($ ) and the SLMA group $(\bullet)$. Values are mean \pm SD. $* \mathrm{P}<0.05$ compared with T2 value in each group. There were no significant differences in peak inspiratory pressure and lung compliance between the groups. T1: 1 min after induction, T2: 5 min before $\mathrm{CO}_{2}$ pneumoperitoneum, T3: 5 min after start of $\mathrm{CO}_{2}$ pneumoperitoneum, T4: 5 min before removal of pneumoperitoneum, T5: 5 min after removal of pneumoperitoneum. 
ies comparing the SLMA and i-gel devices. They concluded that both SADs were similar and had good success rates and short insertion times.

The OLP is commonly measured to quantify the seal with the airway when an SAD is used [12]. It can indicate the success of positive pressure ventilation and the degree of airway protection [14], and it is regarded as the most important value when testing the suitability of an LMA for laparoscopic procedures [15]. The findings of this study are consistent with those of many studies $[11,16,17]$, but they differ from those of another study that reported a higher leak pressure in the LMA Proseal group, with increased airway leak pressure necessitating a better seal than that in the i-gel group [1]. A higher OLP and lower PIP may be advantageous regarding positive pressure ventilation, especially in obese patients, patients with restrictive pulmonary disease, patients undergoing lithotomy or laparoscopic surgery, and in patients of whom the Trendelenburg position is used [18]. In this study, the mean OLP (TO1-3) was higher than the mean PIP (T1, 3, 5) before, during, and after the pneumoperitoneum for both SADs.

Every gastric tube was successfully inserted on the first attempt. However, it was more difficult to insert gastric tubes for patients in the i-gel group because of the smaller aperture of the gastric access port, resulting in a longer insertion time. Some studies $[4,6,19]$ reported that i-gel provided an improved fiberoptic view of glottis compared with that of another SAD, but the outcome of this study was a similar result between the tested SADs.

In the present study, air leakage was detected in five patients in the i-gel group and eight patients in the SLMA group. During pneumoperitoneum, airway manipulations (e.g., pushing and pulling of the device, jaw thrust, chin lift, and neck extension and flexion to maintain adequate ventilation) were performed on four patients in the i-gel and seven patients in the SLMA group, but simple manipulation and re-taping of the device were sufficient. Laparoscopic surgery adversely affects intraoperative pulmonary mechanics, thus providing the most severe test of the efficacy of an airway device [9]. It can also create gastric distension, which may be a cause of nausea and vomiting, as well as perforation of the stomach. Pulmonary compliance is decreased, and the resistance is increased, thereby leading to high airway pressure [20]. Laparoscopic surgery entails increased intraabdominal pressure, typically $15 \mathrm{mmHg}$ [21]. This is associated with an increased peak airway pressure of approximately $50 \%$, which decreases pulmonary compliance by $25 \%[20,22,23]$. Consistently, this study observed decreased compliance and increased peak airway pressure during pneumoperitoneum in both groups, but there were no significant differences in the extent of the changes between the groups. The SADs offer greater hemodynamic stability to insertion, both during maintenance and at extubation, when compared with tracheal tubes [24,25]. In this study, there was a similar trend of changes in the heart rate and blood pressure for both groups.

On removal of the i-gel and SLMA devices, no significant postoperative complications were observed, such as blood on the surface of the device or postoperative sore throat. A sore throat occurred in four patients in the i-gel group and one patient in the SLMA, but this complication is unlikely to be clinically important. These limited data suggest that the flat inflatable cuff of the SLMA device minimizes a sore throat during device insertion. However, Ragazzi et al. [7] reported that a sore throat was more common in the SLMA group than in the i-gel group. The inflatable cuff of the SLMA device can compress terminal nerve endings and microvascular structures in pharyngolaryngeal tissues [26]. There was no incidence of severe airway trauma, such as laryngeal stridor, laryngospasm, bronchospasm, hypoxia, or aspiration.

There are several important limitations to this study. Data were collected by an unblinded investigator, thus, we were unable to ensure that there would not be an element of bias. Secondly, all insertions were performed by a single experienced anesthesiologist; hence, these results may not be applicable to non-experienced users. Nevertheless, Ragazzi et al. [7] reported that both the i-gel and SLMA can be inserted by inexperienced operators with a high rate of successful insertion on the first attempt. Finally, this study used a muscle relaxant before device insertion. The use of neuromuscular blocking drugs can change the laryngeal mask airway leak pressure and may result in a lower measured OLP [27].

Irrespective of the limitations, the study confirmed that both the i-gel and SLMA offer similar performance for laparoscopic cholecystectomy. Compared with the i-gel device, the SLMA device was associated with a shorter gastric tube insertion time. Occasionally, significant complications such as air leakage because of a sudden increase of the intra-abdominal pressure during a laparoscopic operation can occur, and some patients may require airway manipulations to maintain adequate ventilation.

\section{Acknowledgments}

This study was supported by Dong-A University research fund. 


\section{References}

1. Sharma B, Sehgal R, Sahai C, Sood J. PLMA vs. I-gel: A comparative evaluation of respiratory mechanics in laparoscopic cholecystectomy. J Anaesthesiol Clin Pharmacol 2010; 26: 451-7.

2. Jeon WJ, Cho SY, Baek SJ, Kim KH. Comparison of the Proseal LMA and intersurgical I-gel during gynecological laparoscopy. Korean J Anesthesiol 2012; 63: 510-4.

3. Uppal V, Gangaiah S, Fletcher G, Kinsella J. Randomized crossover comparison between the i-gel and the LMA-Unique in anaesthetized, paralysed adults. Br J Anaesth 2009; 103: 882-5.

4. Francksen H, Renner J, Hanss R, Scholz J, Doerges V, Bein B. A comparison of the i-gel with the LMA-Unique in non-paralysed anaesthetised adult patients. Anaesthesia 2009; 64: 1118-24.

5. Uppal V, Fletcher G, Kinsella J. Comparison of the i-gel with the cuffed tracheal tube during pressure-controlled ventilation. Br J Anaesth 2009; 102: 264-8.

6. Theiler LG, Kleine-Brueggeney M, Kaiser D, Urwyler N, Luyet C, Vogt A, et al. Crossover comparison of the laryngeal mask supreme and the i-gel in simulated difficult airway scenario in anesthetized patients. Anesthesiology 2009; 111: 55-62.

7. Ragazzi R, Finessi L, Farinelli I, Alvisi R, Volta CA. LMA Supreme ${ }^{\mathrm{TM}}$ vs i-gel ${ }^{\mathrm{TM}}$ - a comparison of insertion success in novices. Anaesthesia 2012; 67: 384-8.

8. Eschertzhuber S, Brimacombe J, Kaufmann M, Keller C, Tiefenthaler W. Directly measured mucosal pressures produced by the i-gel ${ }^{\mathrm{TM}}$ and laryngeal mask airway Supreme ${ }^{\mathrm{TM}}$ in paralysed anaesthetized patients. Anaesthesia 2012; 67: 407-10.

9. Maltby JR, Beriault MT, Watson NC, Liepert D, Fick GH. The LMA-ProSeal is an effective alternative to tracheal intubation for laparoscopic cholecystectomy. Can J Anaesth 2002; 49: 857-62.

10. Keller C, Brimacombe J, Pühringer F. A fibreoptic scoring system to assess the position of laryngeal mask airway devices. Interobserver variability and a comparison between the standard, flexible and intubating laryngeal mask airways. Anasthesiol Intensivmed Notfallmed Schmerzther 2000; 35: 692-4.

11. Teoh WH, Lee KM, Suhitharan T, Yahaya Z, Teo MM, Sia AT. Comparison of the LMA supreme vs the i-gel in paralysed patients undergoing gynaecological laparoscopic surgery with controlled ventilation. Anaesthesia 2010; 65: 1173-9.

12. Lee JR, Kim MS, Kim JT, Byon HJ, Park YH, Kim HS, et al. A randomised trial comparing the i-gel (TM) with the LMA Classic (TM) in children. Anaesthesia 2012; 67: 606-11.

13. Chen X, Jiao J, Cong X, Liu L, Wu X. A comparison of the performance of the I-gel ${ }^{\mathrm{TM}}$ vs, the LMA-S ${ }^{\mathrm{TM}}$ during anesthesia: a meta-analysis of randomized controlled trials. PLoS One 2013; 8: e71910.

14. Keller C, Brimacombe JR, Keller K, Morris R. Comparison of four methods for assessing airway sealing pressure with the laryngeal mask airway in adult patients. Br J Anaesth 1999; 82: 286-7.

15. Timmermann A, Cremer S, Eich C, Kazmaier S, Bräuer A, Graf BM, et al. Prospective clinical and fiberoptic evaluation of the Supreme laryngeal mask airway. Anesthesiology 2009; 110: 262-5.

16. Cattano D, Ferrario L, Maddukuri V, Sridhar S, Khalil Y, Hagberg CA. A randomized clinical comparison of the Intersurgical i-gel and LMA Unique in non-obese adults during general surgery. Minerva Anestesiol 2011; 77: 292-7.

17. Joly N, Poulin LP, Tanoubi I, Drolet P, Donati F, St-Pierre P. Randomized prospective trial comparing two supraglottic airway devices: i-gel ${ }^{\mathrm{TM}}$ and LMA-Supreme ${ }^{\mathrm{TM}}$ in paralyzed patients. Can J Anaesth 2014; 61: 794-800.

18. Lopez-Gil M, Brimacombe J. The ProSeal laryngeal mask airway in children. Paediatr Anaesth 2005; 15: 229-34.

19. Beringer RM, Kelly F, Cook TM, Nolan J, Hardy R, Simpson T, et al. A cohort evaluation of the paediatric i-gel $\left({ }^{\mathrm{TM}}\right)$ airway during anaesthesia in 120 children. Anaesthesia 2011; 66: 1121-6.

20. Pelosi P, Foti G, Cereda M, Vicardi P, Gattinoni L. Effects of carbon dioxide insufflation for laparoscopic cholecystectomy on the respiratory system. Anaesthesia 1996; 51: 744-9.

21. Versichelen L, Serreyn R, Rolly G, Vanderkerckhove D. Physiopathologic changes during anesthesia administration for gynecologic laparoscopy. J Reprod Med 1984; 29: 697-700.

22. Cunningham AJ, Brull SJ. Laparoscopic cholecystectomy: anesthetic implications. Anesth Analg 1993; 76: 1120-33.

23. O’Malley C, Cunningham AJ. Physiologic changes during laparoscopy. Anesthesiol Clin North America 2001; 19: 1-19.

24. Ismail SA, Bisher NA, Kandil HW, Mowafi HA, Atawia HA. Intraocular pressure and hemodynamic response to insertion of the i-gel, laryngeal mask airway or endotracheal tube. Eur J Anaesthesiol 2011; 6: 443-8.

25. Aydogmus MT, Turk HS, Oba S, Unsal O, Sinikoglu SN. Can Supreme ${ }^{\mathrm{TM}}$ laryngeal mask airway be an alternative to endotracheal intubation in laparoscopic surgery? Braz J Anesthesiol 2014; 64: 66-70.

26. Levitan RM, Kinkle WC. Initial anatomic investigations of the I-gel airway: a novel supraglottic airway without inflatable cuff. Anaesthesia 2005; 60: 1022-6.

27. Goldmann K, Hoch N, Wulf H. Influence of neuromuscular blockade on the airway leak pressure of the ProSeal laryngeal mask airway. Anasthesiol Intensivmed Notfallmed Schmerzther 2006; 41: 228-32. 